\title{
Measuring the Affective and Cognitive Bases of Implicit and Explicit Attitudes Towards Domestic and Foreign Food Brands
}

\author{
Biruta Urbane ${ }^{1}$ Mg. psych.; (DIrina Plotka ${ }^{2}$ Dr. psych. \\ (D) Nina Blumenau ${ }^{3}$ Dr. sc. ing.; Dmitry Igonin ${ }^{4}$ Dr. psych. \\ Baltic International Academy, Latvia

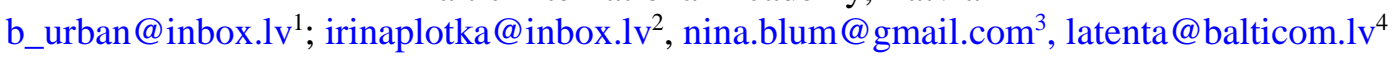

\begin{abstract}
The problem of accounting automatic affective and cognitive processes as bases for implicit attitudes towards brands, as well as methods for measuring them, is poorly developed. An analysis of previous research shows that the study of attitudes towards brands in terms of their affective and cognitive components is mainly carried out using self-assessment procedures. The aim of this research is to measure the affective and cognitive bases of implicit and explicit attitudes towards brands of domestic and foreign foods. Participants $N=131$, aged 17-57 $(M d n=31)$. Measures: specifically designed methodically balanced procedures for measuring implicit and explicit attitudes towards food brands: affective and cognitive implicit associative tests (IAT), Self-Concept IAT; emotional and cognitive explicit procedures and demographic questionnaires. The consistency of the results of implicit and explicit measurements is shown. The results of the measurements of implicit attitudes using three IAT procedures are related too. Using factor analysis, the independence of the constructs of explicit and implicit attitudes towards brands was confirmed, which is interpreted in terms of the theory of double attitudes. The greatest contribution to the implicit attitude, measured by the Self-concept IAT, is made by the cognitive component of attitude, which represents the implicit brand associations of domestic or foreign foods with attributes that characterize the price and quality of the foods. All implicit assessments obtained separately using the affective and cognitive procedures of the IAT, as well as the Self-concept IAT, testified in favour of the preference for foods of domestic brands. However, explicit assessments of the frequency of consumption of the brands under consideration did not reveal preferences for any of them. This discrepancy is seen not only as evidence of a possible ambivalent interaction between affective and cognitive associations, but also as an indication of the importance of future measurements of implicit assessments of instrumental associations that are the result of instrumental learning from consumers. This has the potential to improve the predictive validity of implicit measurements of brand attitudes and to better understand the structure of implicit consumer attitudes and the mechanisms of their influence on behaviour.
\end{abstract}

Keywords: implicit and explicit attitudes towards brand, implicit associative test, attitude's affective and cognitive base, overall attitude, consumer psychology.

\section{Introduction}

The importance of studying consumer attitudes (attitudes towards brands or brand attitudes) lies in the fact that they influence consumer behaviour. Consumer behaviour is highly dependent on both automatic, unconscious cognitive processes and controlled, conscious ones (Perkins, Forehand, 2010; Dimofte, 2010). Accounting of the automatic affective and semantic (cognitive) processes underlying consumer attitudes will contribute to a deeper understanding of how the general representation of attitudes is formed. There is the problem of measuring the affective and cognitive aspects of the explicit and implicit of brand attitudes using direct and indirect measurements.

The modern conceptualization of the attitude is that it represents an overall assessment of an object based on cognitive, affective and behavioural information (Eagly, Chaiken, 1993; Maio, Haddock, Verplanken, 2018). This definition presupposes three substantive components of the attitude, which make up a generalized assessment of the attitude and indicate its general structure.

\section{Implicit and Explicit Attitudes}

In the literature devoted to the issue of studying attitudes, there is a lot of controversy regarding the understanding of the concept of attitudes and the theoretical models that explain their nature. Basically, theoretical models present implicit and explicit attitudes as two qualitatively different ways of cognitive 
information processing (Devine, 1989; Fazio, 1990; Fazio, 1995; Gawronski, Bodenhausen, 2006; Greenwald, Banaji, 1995; Smith, DeCoster, 2000; Strack, Deutsch, 2004; Chaiken, Trope, 1999).

One of them is associative, based on associations of similarity, contiguity and space and time, while the other, a propositional process, operates on the basis of logical analysis and reasoning (Gawronski, Bodenhausen, 2006; Payne, Gawronski, 2010).

It is generally accepted that both explicit and implicit attitudes can be viewed as a result of the learning process. An individual, colliding with objects of the surrounding world, forms an attitude towards them based on the experience of subject-object interactions at the behavioural, cognitive and/or affective level. The results of this interaction are recorded in memory, in the form of assessments of the properties of objects that differ in content and level of generalization, i.e., are the product of explicit or implicit learning (Plotka, Igonin, Blumenau, 2016).

In traditional theories of human learning, the dual nature of its mechanisms is noted. Both mechanisms correspond to two independent and competing systems - associative and propositional. Associations and propositions, as forms of knowledge, differ in the ways of mental representations as well as the conditions under which they are activated and guide behaviour (Mitchell, De Houwer, Lovibond, 2009). These views have contributed to the theoretical understanding of the concept of attitude. Implicit attitudes are viewed primarily as the result of associative processes, and explicit attitudes as propositional (Payne, Gawronski, 2010; Plotka, Igonin, Blumenau, 2016). The process of attitude actualization may require volitional efforts and be conscious, controlled, or, on the contrary, be spontaneous, unconscious or automatic. In the first case, we are talking about explicit attitudes, and in the second - about implicit ones.

R.H. Fazio defined implicit attitudes as "object-evaluation associations in memory" (Fazio, 1995, 247). This understanding of attitude points to associations as a mechanism for the formation of attitudes, and to memory as a storage system for mental representations of attitudes.

Associative assessment (implicit attitudes), in the opinion of some representatives of traditional twoprocess models, is best characterized as automatic affective reactions resulting from the automatic activation of certain associations when one encounters a corresponding relevant stimulus (Gawronski, Bodenhausen, 2006). Such activation processes do not require much cognitive capacity or intention to evaluate an object (Cunningham, Raye, Johnson, 2004).

Further development of knowledge about traditional two-process models, implicit processes, implicit attitudes in social cognition, was due to the achievements in cognitive neuroscience, in particular, the development of the Memory Systems Model (MSM) (Amodio, Mendoza, 2010; Amodio, Ratner, 2011; Amodio, 2019).

\section{Affective and Cognitive Aspects of Implicit Attitude}

Some scholars note that the classical Dual-Process Models consider implicit social processes as singlesystem models of associations among concepts. However, these models do not take into account the entire range of implicit social processes associated with behaviour.

However, the emergence of the MSM model that includes many forms of learning and memory associated with various neural substrates, systems of perception and behaviour (and connections with perceptual and behavioural systems) further influenced a different understanding of implicit social processes (Amodio, Mendoza, 2010; Amodio, 2019).

The MSM model differs in its implicit processing mode from traditional two-process models based on automatic and controlled processes. According to the MSM, implicit processes represent multiple systems representations of associative knowledge, while dual-process models reflect single-system of representations of associative knowledge (Amodio, Ratner, 2011; Amodio, 2019).

Investigating intergroup implicit attitudes (implicit bias) based on the MSM approach (Amodio, Harmon-Jones, Devine, 2003; Amodio, Devine, 2006; Amodio, 2008), researchers found that affective forms of implicit bias correspond to affective forms of learning and memory, which are supported by the amygdala and the associated subcortical circuitry. In contrast, implicit stereotyping reflects semantic associations that correspond to conceptual forms of learning and memory and related areas of the neocortex, such as the left prefrontal cortex (Amodio, Mendoza, 2010). According to MSM, implicit 
assessment (attitudes) can "reflect a combination of affective and semantic (i.e., cognitive) associations". As the researchers note, "MSM will become more common as the field of psychology becomes increasingly interdisciplinary" (Amodio, Mendoza, 2010, 367).

The theoretical implementation of the above stated thesis (on the difference between the affective and cognitive bases of implicit attitudes) will be reflected in our study, which is aimed at studying the cognitive and affective aspects of implicit attitudes toward brand.

Attitude towards Food Brand and Methods of its Measurement

The traditional methods for measuring attitudes are explicit methods. Explicit methods are direct, controlled and conscious (Petty, Fazio, Brinol, 2009).

Traditionally, consumer attitude was conceptualized as a uni-dimensional construct reflecting one's general evaluation of an object (Bagozzi, Burnkrant, 1980; Fishbein, Ajzen, 1975) and is measured using different self-assessment procedures, including different Likert scales (Likert, 1932), semantic differential (Osgood, Suci, Tannenbaum, 1957).

Scientists have identified two aspects of the formation of attitudes towards brands and foods: affective and cognitive (Batra, Ahtola, 1990; Crowley, Spangenberg, Hughes, 1992; Herz, Diamantopoulos, 2013; Voss, Spangenberg, Grohmann, 2003; Crites, Fabrigar, Petty, 1994).

In relation to consumer attitudes, studies by R. Batra and O.T. Ahtola (1990) are often cited. These scholars, citing their predecessors (Holbrook, Hirschman, 1982; Millar, Tesser, 1986; Triandis, 1977), used the idea that consumer attitudes are inherently bidimensional since consumers satisfy both their hedonistic (affective) and utilitarian (cognitive) needs. This thesis has been tested empirically using two measuring scales of semantic differential: hedonistic (affective) and utilitarian (cognitive) to assess overall attitudes subdimensions (Batra, Ahtola, 1990). For example, researchers showed that implicit attitudes towards a country can automatically influence a consumer's cognitive and affective brand perception (Herz, Diamantopoulos, 2013).

Implicit Measurements of Attitudes towards Brands

Well-known experts believe that implicit measures are an important and promising methodological tool, since, to make a consumer choice, it is not enough to take into account only conscious and deliberate processing of information (Dimofte, 2010; Dijksterhuis et al., 2005).

Implicit measures are indirect, automatic, unconscious (Petty, Fazio, Brinol, 2009), and are based on measuring the reaction time of participants whose attention is focused on completing tasks, rather than on the object of attitude (Rudman, 2011).

Implicit measures have a number of advantages over self-assessment procedures, since when performing self-assessment procedures, an individual may either not be aware of his or her opinion of the object of attitude or may not want to express it publicly (Greenwald, Banaji, 1995). Researchers recognize that implicit measurements do not replace but complement the results obtained by explicit measurements (Dimofte, 2010; Dijksterhuis et al., 2005).

The Implicit Association Test (IAT) is most commonly used for measuring consumer attitudes (Schnabel, Asendorpf, Greenwald, 2008; Greenwald et al., 2009; Maison, Greenwald, Bruin, 2004; Friese, Wänke, Plessner, 2006; Priluck, Till, 2010; Dimofte, Yalch, 2011).

\section{Conformity of Explicit and Implicit Measurements}

It should be noted that one of the most important issues in implicit social cognition is the problem of the relationship between explicit and implicit measurements. As the researchers point out, implicit measurements of attitudes (in particular, consumer attitudes) show different results compared to self-assessment procedures.

The results of the meta-analysis show that the relationship between explicit and implicit measurements is often insignificant compared to "sensitive attitudes" such as ethnic ones, towards violence, towards alcohol. However, consumer attitudes studies have shown a higher correlation between implicit and explicit measurements (Greenwald et al., 2009; Perkins et al., 2008). 
Notwithstanding that some researchers question the additional value of implicit measures, the growing number of implicit measurements of brand attitudes refutes this claim (Maison, Greenwald, Bruin, 2001, 2004; Perkins, Forehand, 2010; Schnabel, Asendorpf, Greenwald, 2008; Dimofte, 2010; Maison, Gregg, 2016; Plotka, Urbane, Blumenau, 2015).

Researchers, while studying brand attitudes using the experimental IAT procedure, have generally measured the "undivided" automatic associations as the base of implicit brand attitudes (Maison, Greenwald, Bruin, 2001, 2004. Perkins et al., 2008; Brunel, Tietje, Greenwald, 2004; Dimofte, Johansson, Ronkainen, 2008; Priluck, Till, 2010; Dimofte, Johansson, Bagozzi, 2010; Bosshard et al., 2016; Maison, Maliszewski, 2016).

Thanks to modern advances in cognitive neuroscience in implicit social cognition, it is possible to measure both automatic affective and automatic semantic associations, which are the bases of implicit consumer attitudes (Amodio, Mendoza, 2010). The above conceptualization of implicit brand attitudes has only been applied in some studies of brand and product attitudes (Trendel, Werle, 2015; SarabiaAndreu, Sarabia-Sánchez, Moreno-Albaladejo, 2019). For example, O. Trendel and C.O.C. Werle (2015), in their empirical study, proved that the affective and cognitive bases of implicit attitudes are different structures that independently form an overall implicit attitude toward food. Moreover, each of the bases of the implicit attitude specifically affects the choice of products (food) in certain conditions.

The deeper understanding of the implicit attitude as a two-dimensional structure based on a set of automatic emotional and semantic associations has provided new substantiated opportunities for constructing original versions of the affective IAT, cognitive IAT and Self-Concept IAT (overall) towards the brands of Latvian and foreign foods and the corresponding explicit measuring procedures: affective and cognitive.

The aim of this research is to measure the affective and cognitive bases of implicit and explicit attitudes towards brands of domestic and foreign foods.

\section{Methodology}

The research questions are as follows:

1. Is there a correspondence between the results of implicit and explicit measurements of attitudes towards Latvian or foreign brand foods, measured using the IAT experimental procedures and self-assessment procedures?

2. Is there a relationship between the results of measurements of implicit preferences for brands of Latvian or foreign foods obtained using the affective IAT, cognitive IAT and Self-Concept IAT?

3. Are the results of implicit measurements and explicit measurements independent from each other?

4. What contribution to the implicit attitude, measured with Self-Concept IAT, is made by the emotional and cognitive bases of attitude, measured using the affective IAT, cognitive IAT?

5. How are consumers who demonstrate explicit and implicit preferences for (I) Latvian food brands, (II) foreign food brands and (III) with no preference effect (ambivalent or unexpressed) distributed and how do the results of measurements of explicit attitudes towards food according to the demographic questionnaire relate to each other?

Participants: $N=131$, aged 17-57 years $(M d n=31, M=31.6, S D=9.30)$. Male -42 , female -89 , students and graduate students.

\section{Measures}

\section{Implicit Association Tests}

Two-category Affective and Cognitive Implicit Association Tests (IATs) were designed according to the classic two-category IAT (Greenwald, McGhee, Schwartz, 1998).

- Affective IAT measures the automatic affective associations of Latvian and foreign food brands with attributes having positive or negative valence.

- Cognitive IAT measures the automatic semantic (cognitive) associations of Latvian and foreign food brands with attributes that characterize the price and quality of foods. 
Two-category Self-Concept IAT based on (Greenwald, Farnham, 2000).

- Self-Concept IAT measures automatic associations between categories: "Self” (Me, I, Self, My) and "Others" (Own, Others, They, Them, Your, You) and attributes characterizing Latvian or foreign food brands.

\section{Explicit Procedures}

The basis for the development of self-assessment procedures was the use of some aspects of C.E. Osgood's concept of semantic differential, as well as ideas of modern scholars who applied it to study attitudes toward brands of various food categories (Osgood, Suci, Tannenbaum, 1957; Batra, Ahtola, 1990; Crites, Fabrigar, Petty, 1994; Crowley, Spangenberg, Hughes, 1992; Herz, Diamantopoulos, 2013; Sarabia-Andreu, Sarabia-Sánchez, Moreno-Albaladejo, 2019).

- Affective Explicit Procedure (AEP) measures the explicit preferences of Latvian or foreign food brands, which are based on the assessment of bipolar adjectives characterizing the affective properties of perception of these food brands.

- Cognitive Explicit Procedure (CEP) measures the explicit preferences of Latvian or foreign food brands based on the assessment of bipolar adjectives characterizing the perception of the price and quality of the brands of these foods.

\section{Methodological Balance of Implicit and Explicit Procedures}

The main principle of developing implicit and explicit measuring procedures was their methodological balance, both in the selection of identical Latvian and foreign brands (visual stimuli) and verbal stimuli (adjectives), which are attributes in the affective and cognitive IAT.

- Affective IAT: categories - visual stimuli (Figure 1), attributes - affective verbal stimuli (Table 1).

- Cognitive IAT: categories - visual stimuli (Figure 1), attributes - cognitive verbal stimuli (Table 1).

- Affective Explicit Procedure (AED): visual stimuli (Figure 1), affective verbal stimuli (Table 1).

- Cognitive Explicit Procedure (CEP): visual stimuli (Figure 1), cognitive verbal stimuli (Table 1).

- Self-concept IAT: categories - Self, Others, attributes - visual stimuli (Figure 1).
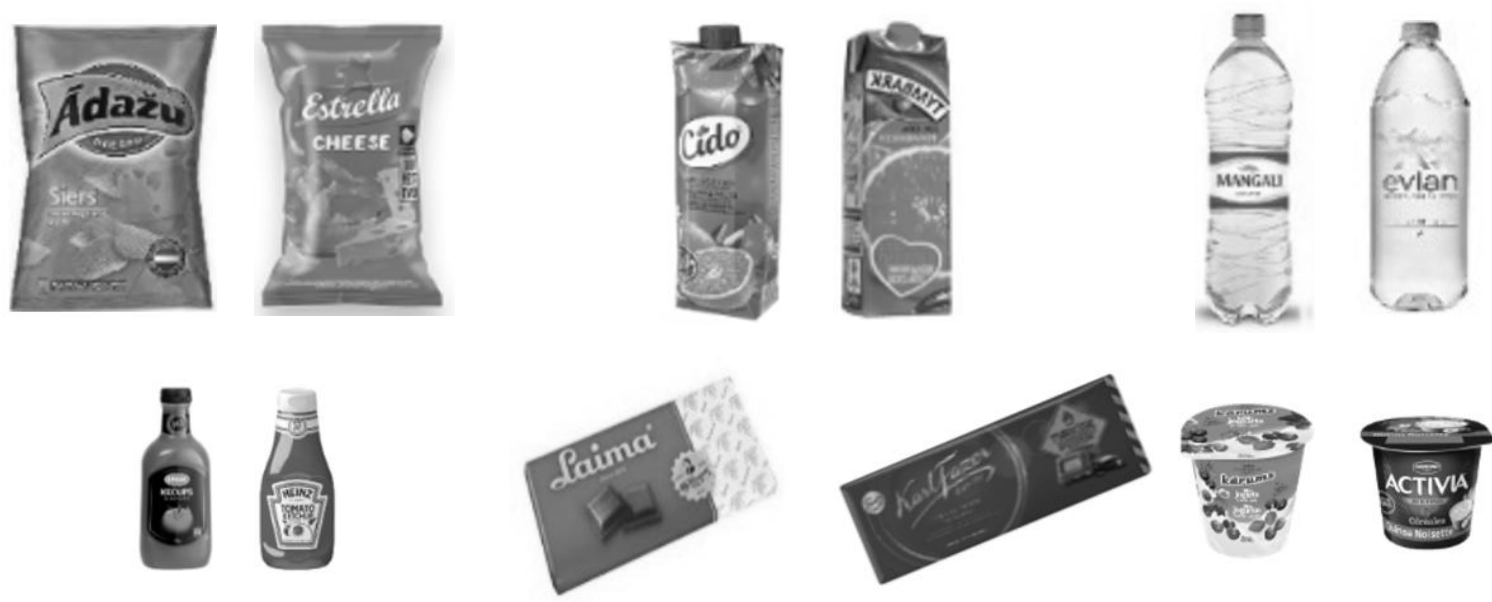

Figure 1. Visual stimuli.

Visual stimuli (Figure 1). The brands of Latvian and foreign foods were selected based on the results of interviews with representatives of supermarkets, who reported on the most popular categories of foods that consumers most often use in the Latvian market. To prevent the participants from focusing on only one category of food brands, the experiment included brands of Latvian and foreign foods from different categories (yoghurts, chocolate, juices, ketchup, mineral water, chips). Visual stimuli (product brands) were selected for similarity, size, visual complexity and image resolution (Rothermund, Wentura, 2004). 12 visual stimuli contain 6 images of Latvian food brands (chips "Ādažu čipsi"; mineral water "Mangal̦u ūdens"; juice "Cido"; ketchup "Spilva"; chocolate "Laima", yogurt "Kārums") and 6 images with brands of foreign foods (chips "Ekstrella"; mineral water "EVIAN", juice "TYMBARK"; ketchup "Heinz"; chocolate "Karl Fazer", yogurt "ACTIVIA"). Visual stimuli were colour images. 
Verbal stimuli (Table 1). The basis for the development of self-assessment procedures was the use of some aspects of the concept of semantic differential (Osgood, Suci, Tannenbaum, 1957), as well as the ideas of modern scholars who applied this concept to study the attitudes to brands and goods, both in hedonic and utilitarian aspects (Batra, Ahtola, 1990; Crites, Fabrigar, Petty, 1994; Crowley, Spangenberg, Hughes, 1992; Herz, Diamantopoulos, 2013; Sarabia-Andreu, Sarabia-Sánchez, MorenoAlbaladejo, 2019). The selection of attributive bipolar adjectives was based on the consideration of the two factors "Assessment" and "Strength" of C.E. Osgood's concept (Osgood, Suci, Tannenbaum, 1957).

Table 1

Verbal stimuli: bipolar adjectives

\begin{tabular}{|l|l|l|}
\hline \multicolumn{1}{|c|}{ Adjectives } & \multicolumn{1}{|c|}{ Positive } & \multicolumn{1}{|c|}{ Negative } \\
\hline Affective & $\begin{array}{l}\text { pleasant, alive, fancied, aromatic, tasty, } \\
\text { healthy }\end{array}$ & $\begin{array}{l}\text { unpleasant, dead, disgusting, smell, not tasty, } \\
\text { unhealthy }\end{array}$ \\
\hline Cognitive & $\begin{array}{l}\text { economical, profitable, convenient, effective, } \\
\text { natural, safe }\end{array}$ & $\begin{array}{l}\text { uneconomical, unprofitable, inconvenient, } \\
\text { ineffective, chemical, unsafe }\end{array}$ \\
\hline
\end{tabular}

For affective bipolar adjectives: the "Assessment" factor corresponds to pairs: pleasant / unpleasant, fancied / disgusting, aromatic / smelly, tasty / tasteless, and the "Strength" factor corresponds to bipolar adjectives: healthy / unhealthy, alive / dead.

For cognitive bipolar adjectives: the "Assessment" factor corresponds to the pairs: economical / uneconomical, profitable / disadvantageous, effective / ineffective, convenient / inconvenient, and the "Strength" factor corresponds to bipolar adjectives: natural / chemical, safe / unsafe.

We also found similar adjectives in studies measuring the affective component of brand attitudes: pleasant / unpleasant, nice / awful (Batra, Ahtola, 1990), pleasant / unpleasant, love / hateful (Crites, Fabrigar, Petty, 1994); pleasant / unpleasant, nice / awful (Crowley, Spangenberg, Hughes, 1992); beneficial / harmful (Sarabia-Andreu, Sarabia-Sánchez, Moreno-Albaladejo, 2019); tasty / untasty (Trendel, Werle, 2015); aromatic / stinky, alive - dead (Dalton et al., 2008). In studies that measure the cognitive component of brand attitudes: beneficial / harmful (Batra, Ahtola, 1990); safe / unsafe (Crites, Fabrigar, Petty, 1994); effective / ineffective (Voss, Spangenberg, Grohmann, 2003); natural / chemical, safe / dangerous (Dalton et al., 2008); beneficial / harmful (Crowley, Spangenberg, Hughes, 1992).

\section{Questionnaires}

- A specifically developed a preliminary questionnaire, the purpose of which was to find out how familiar the research participants are with the logos of Latvian and foreign brands and their foods.

- A specifically developed a demographic questionnaire, the purpose of which was to find out gender, age, marital status, education, occupation, income per family member per month (gross, euros) and attitudes towards Latvian and foreign food brands that participants use daily.

\section{IAT Experimental Procedures}

The experimental procedures for the affective and cognitive IATs are shown in Table 2. The experimental procedure for the Self-Concept IAT is shown in Table 3.

Each of the three experimental IAT procedures (Affective, Cognitive and Self-Concept) has seven blocks. Before the start of the experiment on a computer monitor in each of the three IAT procedures, the participant was given general instructions and specific instructions before each of the blocks. The instructions were written in black letters on a white background and positioned in the centre of the screen. Each sentence began on a new line. Each target attribute appeared cantered on the screen. All stimulus words are represented by lowercase letters. The participants' task was to categorize the stimuli presented. The stimuli were displayed on the screen without sound and remained on the screen until the participant pressed the key. One IAT procedure took on average 20 to 25 minutes. The participant's reaction time (RT) was recorded. For each trial, RT was recorded as the time interval between the start of the presentation of the target attribute and the pressing of the correct key. The stimuli were presented at random. With the correct categorization, the subsequent visual and verbal stimulus appeared on the screen after $400 \mathrm{~ms}$. In case of incorrect categorization, a red cross appears on the screen, which automatically 
disappears. Time of stimulus presentation, intervals between stimuli, number of stimuli - words, font and colour background settings did not change. Before data processing, trials with latencies over $5000 \mathrm{~ms}$ were deleted. Also excluded were those participants with more than $10 \%$ of trials showing latencies less than $300 \mathrm{~ms}$. D-scores were used as the size of the IAT effect, the calculation of which was performed in a standard way, which is described, for example, in the book by L.A. Rudman (2011).

Table 2

Affective and cognitive IAT procedures

\begin{tabular}{|l|l|l|l|l|}
\hline Block & Trials & \multicolumn{1}{|c|}{ Function } & \multicolumn{1}{|c|}{ Left-key response “Q” } & \multicolumn{1}{|c|}{ Right- key response “P” } \\
\hline 1 & 24 & Practice & LAT (v) & F (v) \\
\hline 2 & 24 & Practice & Positive (a) & Negative (a) \\
\hline 3 & 48 & Test & LAT (v)+Negative (a) & F (v)+Positive (a) \\
\hline 4 & 48 & Test & LAT (v)+ Negative (a) & F (v)+ Positive (a) \\
\hline 5 & 24 & Practice & F (v) & LAT (v) \\
\hline 6 & 48 & Test & F (v)+Negative (a) & LAT (v)+ Positive (a) \\
\hline 7 & 48 & Test & F (v)+Negative (a) & LAT (v)+ Positive (a) \\
\hline
\end{tabular}

Note. $v$ - visual category, $a$ - adjectives. LAT - Latvian Brand, $F-$ Foreign Brand.

Table 3

Self-Concept IAT

\begin{tabular}{|l|l|l|l|l|}
\hline Block & Trials & Function & Left-key response "Q" & Right- key response "P" \\
\hline 1 & 10 & Practice & Others (w) & Self $(w)$ \\
\hline 2 & 15 & Practice & F (v) & LAT (v) \\
\hline 3 & 40 & Test & Others $(w)+F(v)$ & Self $(w)+L A T(v)$ \\
\hline 4 & 45 & Test & Others $(w)+F(v)$ & Self $(w)+L A T(v)$ \\
\hline 5 & 20 & Practice & I (w) & Not I (w) \\
\hline 6 & 40 & Test & Self $(w)+F(v)$ & Others (w) +LAT (v) \\
\hline 7 & 45 & Test & Self $(w)+F(v)$ & Others (w) +LAT (v) \\
\hline
\end{tabular}

Note.Target categories (verbal stimuli, w): Me, I, Self, My, Own, Others, They, Them, Your, You. Target attributes $\mathrm{v}-$ visual stimuli.

\section{Affective and Cognitive Explicit Procedures}

Affective Explicit Procedure (AEP) and Cognitive Explicit Procedure (CEP) have the same visual stimuli (Figure 1) and different sets of verbal stimuli - adjectives (Table 1). Each of the self-assessment procedures includes 12 questionnaires - according to the number of corresponding adjectives. Each questionnaire contains one adjective from the corresponding column of Table 1 and six pairs of food brands - one Latvian food brand and one foreign food brand. An example of a questionnaire with the adjective "tasty" for the AEP procedure is shown in Table 4.

Instructions to the participant. "Dear participant! Your task is to assess your attitude towards Latvian or foreign food brands by associating them with the specified adjective, for example, "tasty". For each of the pairs of foods of the Latvian and foreign brands, you need to select only one number that corresponds to your assessment and put it in a circle. The numbers " 3 ", "2", "1", " 0 " on the left refer to brands of Latvian foods. The numbers " 0 ", " 1 ", " 2 ", " 3 " on the right refer to brands of foreign foods. Scores denote brand compliance with the specified adjectives " 3 " - great match, " 2 " - medium match, " 1 " small match, "0" - I don't know or no match.

Processing of questionnaires. Scores " 3 ", “2”, "1", “0”, “1”, “2”, “3” are converted as follows. For "good

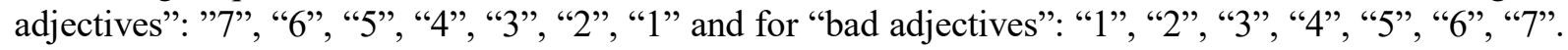
For each participant, the scores for all words are summed up and divided by 72 , since there are 12 adjectives and six pairs of brands: 6 × $12=72$. The result of the division is called the E-score.

Interpretation. Based on the results of the explicit tests, it is possible to determine the explicit preferences of the participant's brands. The main idea of determining preference is shown in Table 5. This table is 
a fragment of a large table, which presents all possible ratings of participants for one of six pairs of brands: Latvian - LV and foreign - F, and for one of six pairs of opposite adjectives: "delicious - tasteless". Table 5 shows seven cases out of 46. Based on the analysis of all cases, it was decided to accept the following interpretation for the E-scores.

Table 4

Affective explicit procedure questionnaire. Adjective: "Delicious"

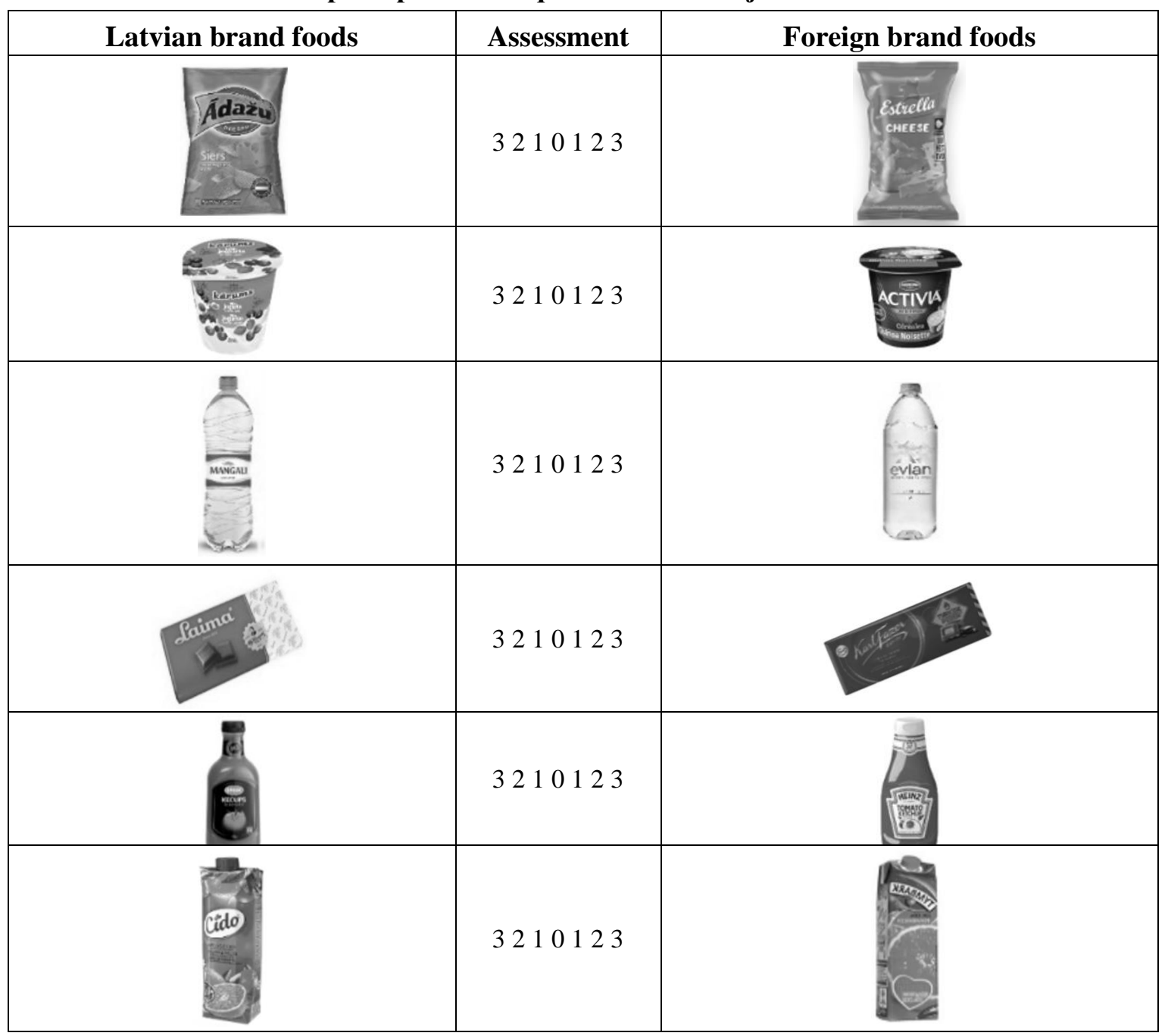

E-scores $\geq 4.5$ correspond to the explicit preferences of Latvian foods. E-scores $\leq 3.5$ correspond to the explicit preferences of foreign brand foods. $3.5<E$-scores $<4.5$ correspond to the absence of the effect of explicit preferences of foreign or Latvian brand foods. Note: Explicit preferences: affective for the AEP test and cognitive for the CEP test.

The internal consistency index of the AEP of the Cronbach's Alpha test is $\alpha=0.91$, which indicates excellent consistency of scale. The internal consistency score of the Cronbach's Alpha test is $\alpha=0.88$, which indicates good internal consistency of the scale.

\section{Demographic Questionnaire}

The demographic questionnaire contained the following information about the participants: gender, age, marital status, education, occupation, income per family member per month (questions 1-6) and attitudes towards Latvian and foreign foods that participants use daily (questions 7-13); what foods (Latvian or foreign) are bought in the first instance; when was the last time Latvian foods were bought; when was the last time foreign foods were bought; which foods (Latvian or foreign) are preferred: a) at an equivalent price, b) with an equivalent quality; whether Latvian foods are recommended to friends and acquaintances and how often; what reason can induce the transition from Latvian foods to foreign foods? 
An example of possible assessments of participants in one pair of Latvian (LV) and foreign (F) brands for a pair of adjectives "tasty-not tasty"

\begin{tabular}{|c|c|c|c|c|c|c|}
\hline \multicolumn{2}{|c|}{$\begin{array}{l}\text { Marked scores (bold) and } \\
\text { transcoding }\end{array}$} & \multicolumn{2}{|c|}{ Tasty - Not tasty } & \multicolumn{2}{|c|}{$\begin{array}{l}\text { Assessment's level of } \\
\text { conformity between } \\
\text { adjectives and brands }\end{array}$} & \multirow[t]{2}{*}{$\begin{array}{l}\text { Preferred } \\
\text { brand }\end{array}$} \\
\hline Tasty & Not tasty & Total & Mean & Tasty & Not tasty & \\
\hline 3210123 & 3210123 & \multirow{2}{*}{14} & \multirow{2}{*}{7} & \multirow{2}{*}{ LV large } & \multirow{2}{*}{ F large } & \multirow{2}{*}{ LV } \\
\hline 7654321 & 1234567 & & & & & \\
\hline 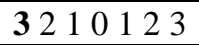 & 3210123 & \multirow{2}{*}{13} & \multirow{2}{*}{6.5} & \multirow{2}{*}{ LV large } & \multirow{2}{*}{ F medium } & \multirow{2}{*}{ LV } \\
\hline 77654321 & 1234567 & & & & & \\
\hline 3210123 & 3210123 & \multirow{2}{*}{12} & \multirow{2}{*}{6} & \multirow{2}{*}{ LV large } & \multirow{2}{*}{ F small } & \multirow{2}{*}{ LV } \\
\hline 7654321 & 1234567 & & & & & \\
\hline 3210123 & 3210123 & \multirow{2}{*}{11} & \multirow{2}{*}{5.5} & \multirow{2}{*}{ LV large } & \multirow{2}{*}{ no preference } & \multirow{2}{*}{ LV } \\
\hline 7654321 & 1234567 & & & & & \\
\hline 32210123 & 3210123 & \multirow{2}{*}{10} & \multirow{2}{*}{5} & \multirow{2}{*}{ LV large } & \multirow{2}{*}{ LV small } & \multirow{2}{*}{ LV } \\
\hline 7654321 & 1234567 & & & & & \\
\hline 3210123 & 3210123 & \multirow{2}{*}{9} & \multirow{2}{*}{4.5} & \multirow{2}{*}{ LV large } & \multirow{2}{*}{ LV medium } & \multirow{2}{*}{ no preference } \\
\hline 7654321 & 1234567 & & & & & \\
\hline 3210123 & 3210123 & \multirow{2}{*}{8} & \multirow{2}{*}{4} & \multirow{2}{*}{ LV large } & \multirow{2}{*}{ F large } & no preference \\
\hline 7654321 & 1234567 & & & & & \\
\hline
\end{tabular}

Procedure. First, the participants filled out the questionnaires (preliminary and demographic). Then they performed Implicit Association Tests, after which - explicit procedures: affective and cognitive. Ninety - four participants performed the experimental procedure for Affective IAT. Ninety - five participants performed the experimental Cognitive IAT procedure. One hundred and two participants performed the experimental procedure Self-Concept IAT. Sixty - six participants performed all three IAT procedures. Explicit procedures were followed by all participants. The researchers followed guidelines for confidentiality, voluntary participation, and psychological ethics.

Apparatus: Certified licensed software E-Prime 2®.

\section{Results and Discussion}

\section{Implicit Variables}

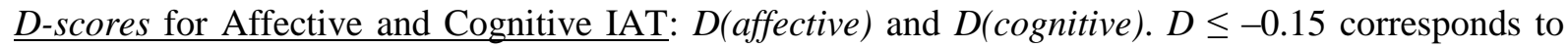
positive associations with Latvian brands and negative ones - with foreign brands, $D \geq 0.15$ corresponds to positive associations with foreign brands and negative ones with Latvian brands, $-0.15<D<0.15-$ no effect.

D-scores for Self-concept IAT: $D($ Self $) \leq-0.15$ corresponds to implicit association of oneself with foreign brands and others - with Latvian brands, $D($ Self $) \geq 0.15$ corresponds to implicit association of oneself with Latvian brands and others with foreign brands, $-0.15<D(S e l f)<0.15$ - no effect.

\section{Explicit variables}

$E$-scores for affective and cognitive explicit tests: E(affective) and $E$ (cognitive). If $E \geq 4.5$ - explicit preferences of Latvian brand foods, with $E \leq 3.5$ - explicit brand preferences for foreign foods.

Demographic variables: Age. $M d n=31.0$. Gender $(1-$ male, 0 - female). Education $(1-$ Secondary, 2 - Student, 3 - Bachelour, 4 - Master). Family status (1 - unmarried, 2 - married). Occupation ( 1 - works, 2 - does not work). Monthly Income per Person $(\epsilon)(1-$ " $<500 ", 2$ - "500-700", 3 - "701900", 4 - "901-1000", 5 - "> 1000"). Preferred Foods (1 - Latvian Foods, 2 - no preference, 3 - Foreign Foods). Last Purchase of Latvian Brand Foods (1 - today, 2 - yesterday, 3 a week ago, 4 - a month ago). Last Purchase of Foreign Brand Foods (1 - today, 2 - yesterday, 3 a week ago, 4 - a month ago). Preference at equal price (1 - Latvian Foods, 2 - no preference, 3 - Foreign Foods). Preference at equal 
quality (1 - Latvian Foods, 2 - no preference, 3 - Foreign Foods). Latvian brand recommendation to friends (1 - no, 2 - yes). The frequency of recommendations of the Latvian brand (1 - never, 2 -sometimes, 3 - often). The reason for the change of foods of Latvian brands to foreign ones (1-Recommendations, 2 - Quality, 3 - Experience, 4 - Price, 5 - The need for diversity).

The study of the data showed that the data distribution allows the use of parametric statistics methods. Methods of statistical data processing were chosen in accordance with the research questions.

Correlation coefficients. The Pearson's $(r)$ and Spearman's $\left(r_{S}\right)$ correlation coefficients were used. The results are shown in the Table 6 and Figure 2.

Table 6

Correlation coefficients between the variables

\begin{tabular}{|l|l|c|c|c|}
\hline \multicolumn{2}{|c|}{ Variables } & Correlation Coefficients & Effect Size & $\begin{array}{c}\text { 95\% Confidence } \\
\text { Interval }\end{array}$ \\
\hline$D$ (affective) & E(affective) & $r_{S}(94)=-0.20, p=0.057$ & between small and medium & {$[-0.38 ; 0.01]$} \\
\hline$D($ cognitive $)$ & $E$ (cognitive) & $r(94)=-0.34, p=0.001$ & medium & {$[-0.51 ;-0.15]$} \\
\hline$D($ self $)$ & E(affective) & $r_{S}(102)=0.26, p=0.009$ & medium & {$[0.065 ; 0.43]$} \\
\hline$D($ self $)$ & E(cognitive) & $r_{S}(102)=0.30, p=0.002$ & medium & {$[0.12 ; 0.47]$} \\
\hline$D($ self $)$ & $D$ (affective) & $r(66)=-0.29, p=0.020$ & medium & {$[-0.50 ;-0.052]$} \\
\hline$D($ self $)$ & $D$ (cognitive) & $r(66)=-0.31, p=0.013$ & medium & {$[-0.51 ;-0.073]$} \\
\hline$D($ affective $)$ & $D$ (cognitive) & $r(66)=0.49, p<0.001$ & large & {$[0.28 ; 0.65]$} \\
\hline
\end{tabular}

Note. Correspondence between the results of implicit and explicit measurements of brand attitudes, measured using experimental procedures of affective and cognitive IAT and explicit affective and cognitive procedures, is available with negative correlation coefficients. Correspondence between the results of implicit and explicit measurements of brand attitudes, measured using the Self-Concept IAT and explicit affective and cognitive procedures, is available with positive correlation coefficients. The relationship between the results of measurements using affective and cognitive IAT, with the results of measurements using the Self-Concept IAT, is available with negative correlation coefficients.

To answer the first research question "Is there a correspondence between the results of implicit and explicit measurements of attitudes towards Latvian or foreign brand foods, measured using the IAT experimental procedures and self-assessment procedures?" the Pearson's $(r)$ and Spearman's $\left(r_{s}\right)$ correlation coefficients were used (Table 6, Figure 2). There is a correspondence between the results of measurements of affective and cognitive implicit and explicit attitudes towards the brand of Latvian or foreign foods. The effect size is medium.

For the results of measurements of cognitive implicit and cognitive explicit attitudes there is a stronger correspondence than for the results of measurements of affective implicit and affective explicit attitudes: $Z=1.37, p=0.086$. There is a trend towards significance, the Cohen's effect size $q=0.20$ is between small and medium.

Partial Correlation coefficients were used to study the influence of the controlled variables Gender, Age, Education, Family Status, Occupation, Monthly Income per Person $(\epsilon)$ on the relationship between the results of implicit and explicit measurements. The relationships between the variables $D$ (cognitive) $E$ (cognitive), $D($ self $)-E$ (affective) and $D($ self $)-E$ (cognitive) are not affected by any of the controlled variables. The relationship between variables $D$ (affective) -E(affective) is affected only by the variable Age.

In the older group of participants (over $M d n=31$ years old), there is a trend towards a statistically significant relationship with the medium effect size: $r_{s}(35)=-0.30, p=0.077$. In the younger group of participants (under $M d n=31$ years old), the relationship was not found: $r(59)=-0.080, p=0.55$, ns. Figure 2 shows the scatterplots for the variables $D$ (affective) - E(affective), where the points with the corresponding regression lines for the younger and older groups of participants are highlighted. 


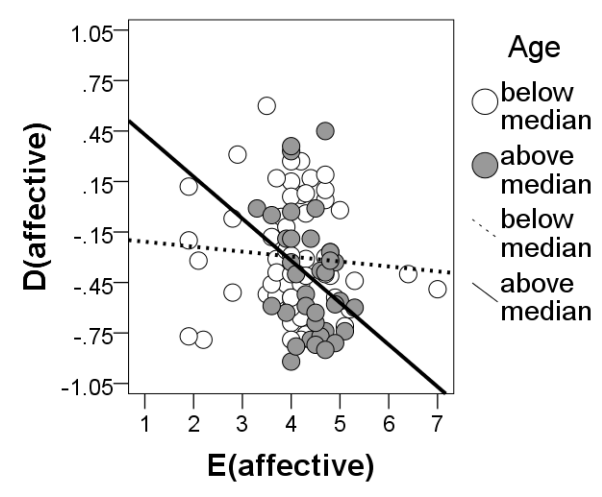

Figure 2. Scatterplots: affective implicit and affective explicit attitudes - correspondence of measurement results for two age groups $(N=94)$.

To answer the second research question "Is there a relationship between the results of measurements of implicit preferences for brands of Latvian or foreign foods obtained using the affective IAT, cognitive IAT and Self-Concept IAT?" the Pearson's $(r)$ and Spearman's $\left(r_{s}\right)$ correlation coefficients were used (Table 6). The results of measurements of implicit attitudes using affective IAT, cognitive IAT with Self-concept IAT are related. The effect size is medium. The results of measurements of the affective IAT and cognitive IAT are related. The effect size is large.

The relationships between the results of these measurements are not affected by any of the controlled variables (Gender, Age, Education, Family Status, Occupation, Monthly Income per Person).

To answer the third research question: "Are the results of implicit measurements and explicit measurements independent from each other?" the factor analysis with two factors (Kaiser criterion) was used (Principal Component Method, Rotation's method Varimax with Kaiser Normalization. Rotation converged in 3 iterations (Table 7, Rotated Component Matrix). Cumulative percent of total variance explained $72.4 \%$, two factors: $37.8 \%+34.6 \%$ ). Scores based on method "Regression" (Table 7, Component Score Coefficient Matrix). Kaiser-Meyer-Olkin Measure of Sampling Adequacy $K M O=0.56$ (almost satisfactory adequacy of the sample). Bartlett's Test of Sphericity (10) $=101$, $p<0.001$ (data are suitable for factor analysis).

As a result (Table 7), it was found that Component 1 is described only by explicit variables E(cognitive), $E$ (affective) and the second Component 2 is described only by implicit variables $D$ (affective), $D$ (cognitive), $D($ self). The Component 1 can be called the "Explicit overall brand attitude" and the Component 2 can be called the "Implicit overall brand attitude".

Table 7 (Component Score Coefficient Matrix) also shows the $\beta$-coefficients for the standardized regression equations with the dependent variables "Explicit overall brand attitude" and "Implicit overall brand attitude". Statistical significance of $\beta$-coefficients $p<0.001$.

Large positive values of the variable "Explicit overall brand attitude" correspond to the preference for the Latvian brand, and large negative values in absolute terms correspond to the preference for a foreign brand. Large positive values of the variable "Implicit overall brand attitude" correspond to the preference of a foreign brand, and large negative values in absolute value correspond to the preference of the Latvian brand.

Table 7

Principal Components Analysis Matrices: Rotated Component Matrix and Component Score Coefficient Matrix

\begin{tabular}{|l|r|r|r|r|}
\hline & \multicolumn{2}{|c|}{ Rotated Component Matrix } & \multicolumn{2}{c|}{ Component Score Coefficient Matrix } \\
\cline { 2 - 5 } & Component 1 & Component 2 & Component 1 & Component 2 \\
\hline$E$ (cognitive) & $\mathbf{0 . 9 4 6}$ & -0.026 & $\mathbf{0 . 5 0 6}$ & 0.049 \\
\hline (affective) & $\mathbf{0 . 9 3 8}$ & -0.090 & $\mathbf{0 . 4 9 7}$ & 0.011 \\
\hline$D($ affective $)$ & 0.190 & $\mathbf{0 . 8 2 9}$ & 0.158 & $\mathbf{0 . 4 9 9}$ \\
\hline$D($ cognitive $)$ & -0.273 & $\mathbf{0 . 7 8 8}$ & -0.093 & $\mathbf{0 . 4 4 4}$ \\
\hline$D($ self $)$ & 0.081 & $\mathbf{- 0 . 6 4 4}$ & 0.000 & $\mathbf{- 0 . 3 7 2}$ \\
\hline
\end{tabular}


All explicitly measured brand attitudes are associated with each other. All implicitly measured brand attitudes are also associated with each other (Table 6). The result of factor analysis is not unexpected. The variables "Explicit overall brand attitude" and "Implicit overall brand attitude" constructed using factor analysis are independent: the correlation between them is equal to zero. The greatest impact on "Explicit overall brand attitude" is made by the variable E(cognitive), then by E(affective). The greatest impact on "Implicit overall brand attitude" is made by the variable $D$ (affective), then by $D$ (cognitive), then by $D($ self $)$. The hypothesis can be put forward that "overall" implicit and "overall" explicit attitudes toward food brands are independent constructs. At the same time, the implicit and explicit cognitive and affective components of attitudes and implicit attitudes measured using the Self-concept IAT are related and, therefore, can reflect two sides of the dual process. Within the framework of models of dual processes, this contradiction can be explained by the influence of propositional processes at an explicit level, which limit or facilitate the manifestation of implicit attitudes.

To answer the fourth research question: "What contribution to the implicit attitude, measured with Self-Concept IAT, is made by the emotional and cognitive bases of attitude, measured using the affective IAT, cognitive IAT?" The multiple regression analysis was used. Dependent variable $D$ (self), independent variables: $D$ (affective) and $D$ (cognitive).

Method: Backward, criterion: Probability of $F$-to-remove $>=0.100$. The equation for estimation:

$$
D(\text { self }) \text { [estimate] }=0.330-0.251 * D(\text { cognitive }) \text {. }
$$

The Beta coefficient is $\beta=-0.31, t(64)=-2.57, p=0.013$. Effect size is medium. 95\% CI $=[-0.093 ; 0.31]$. The statistical significance of intercept is: $t(64)=6.70, p<0.001$. The statistical significance of Model 1 is: $F(1,64)=6.60, p=0.013$. $R$-Square $=0.094$ shows that $9.4 \%$ of variability of the dependent variable $D($ self $)$ is due to the influence of the independent variable $D$ (cognitive). Effect size $R$-Square is between small and medium, 95\% CI $=[-0.036 ; 0.22]$. Effect size Cohen's $f$-Square $=0.10$ is between small and medium, 95\% CI $=[-0.036 ; 0.28]$. Adjusted $R$-square $=0.079$. Standard error of the estimate is 0.31 .

Thus, the greatest contribution to the implicit attitude, as measured by the Self-concept IAT, is made by the cognitive component of the attitude, which represents the implicit brand associations of Latvian foreign foods with attributes that characterize the price and quality of the foods.

The study (Trendel, Werle, 2015) considers the construction of an "overall" implicit attitude to food, which has two independent bases - cognitive and affective. Overall attitude, attitude's cognitive base, and attitude's affective base were defined as the results of measurements using three IAT procedures that had the same categories: "chocolate" and "apple". The attributes were different. The "overall" attitude is built using the IAT procedure, in which the attributes are affectively coloured words. Attributes of the affective IAT were affectively coloured words reflecting taste. Attributes of the cognitive IAT were the choice to use healthy-non-healthy foods. The measure of the "overall" attitude was chosen as the dependent variable. The measures of the cognitive base of the attitude and the affective base of the attitude were chosen as predictors and were independent of each other.

In the present research, the possibility to take the attitude towards food, measured by the Self-Concept IAT as the "overall" attitude was considered. However, only the cognitive basis of the implicit attitude was included into the regression equation. Therefore, a different approach was taken to create an "overall" implicit attitude, namely using factor analysis was taken. The use of factor analysis led to an important result: to obtain "overall" explicit and "overall" implicit attitudes to food brands.

To answer the fifth research question: "How are consumers who demonstrate explicit and implicit preferences for (I) Latvian food brands, (II) foreign food brands and (III) with no preference effect (ambivalent or unexpressed) distributed and how do the results of measurements of explicit attitudes towards food according to the demographic questionnaire relate to each other?" the frequency analysis was used: " $\varphi$ *Fisher's Angular Transformation Test", Pearson's Chi-Square $\left(\chi^{2}\right)$ Test of Independence, Goodness-of-Fit $\left(\chi^{2}\right)$ Test (Figures 3-4). Distributions of nominal variables $D$ (affective), $D$ (cognitive), $D($ self), E(affective), E(cognitive) and Preferred Foods were studied with three categories each: "Latvian brand", "foreign brand" and "no effect". The term "no effect" refers to either very weak or ambivalent preferences. 

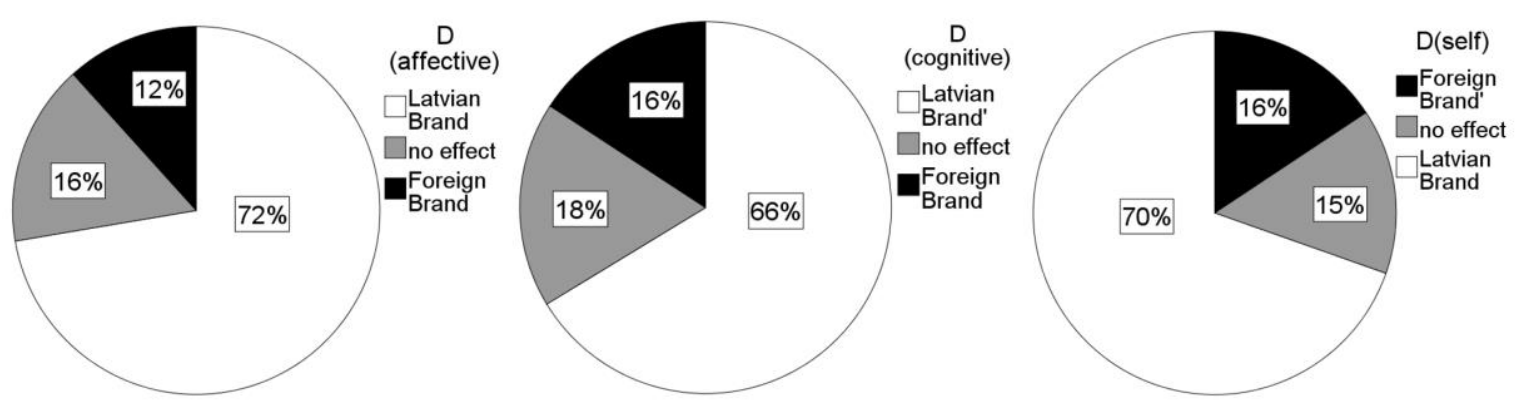

Figure 3. Distribution of implicit attitudes towards brands of Latvian or foreign foods, measured using Affective IAT - D(affective), Cognitive IAT - D(cognitive), and Self-concept IAT - $D$ (self).
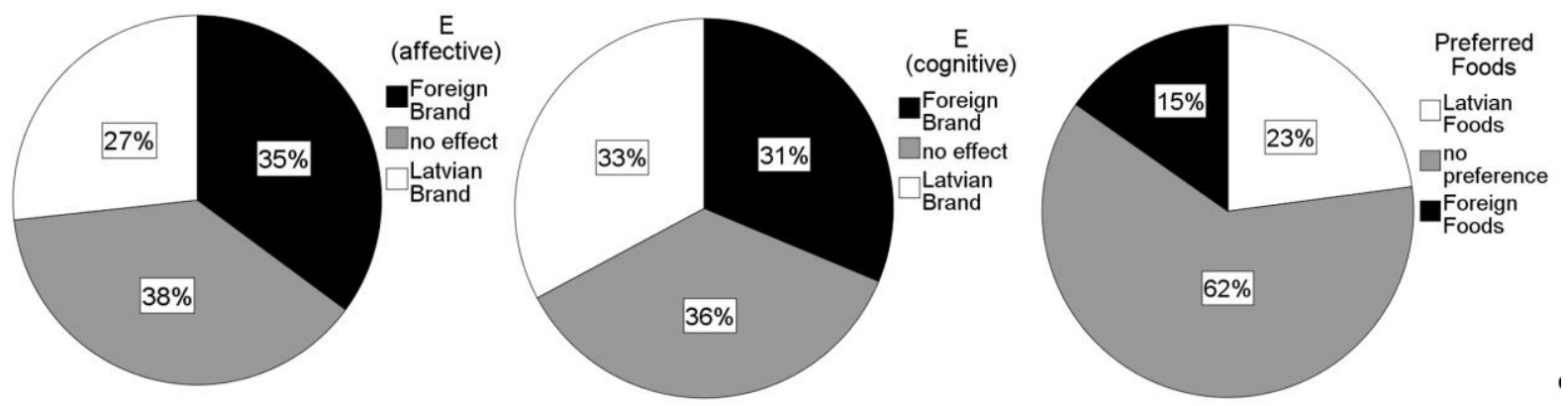

Figure 4. Distribution of explicit attitudes towards brands of Latvian or foreign foods, measured using Affective $-E$ (affective) and Cognitive $-E$ (cognitive) explicit tests, as well as preferences of Latvian or foreign Preferred Foods.

The distributions of the values of all implicit variables are statistically significantly different from the uniform distribution. The size of the effect $\omega$ is large in all cases: $D$ (emotional) $-\chi^{2}(2, N=94)=64.6, p<0.001$, $\omega=0.83 ; D$ (cognitive $)-\chi^{2}(2, N=95)=46.6, p<0.001, \omega=0.70 ; D($ self $)-\chi^{2}(2, N=102)=60.4, p<0.001$, $\omega=0.77$. The largest percentage of participants for all three variables has implicit associations with a Latvian food brand. The size of the Cohen's $h$ effect is large: $D$ (emotional) $-\varphi^{*}=8.31, p<0.001, h=1.21$; $D$ (cognitive) $-: \varphi^{*}=7.10, p<0.001, h=1.03 ; D($ self $)-\varphi^{*}=8.28, p<0.001, h=1.16$. There was no statistically significant difference in the percentage of participants with an implicit preference for a foreign brand and those with no effect. A statistically significant difference in the percent of participants with an implicit preference for foreign brands and participants "without effect" was not revealed.

A statistically significant difference between the distributions of the values of the explicit variables and the uniform distribution was not found: $E$ (emotional) $-\chi^{2}(2, N=131)=2.76, p=0.25, \omega=0.15$, the size of the effect $\omega$ is small. E(cognitive) $-\chi^{2}(2, N=131)=.43, p=0.81, \omega=0.057$, no effect was found. The percentage of participants without an explicit emotional brand preference (38\%) is statistically significantly higher than the percentage of participants with a preference for a Latvian food brand (27\%): $\varphi^{*}=1.99, p=0.047, h=0.25$. The size of the Cohen's $\mathrm{h}$ effect is small. There were no other differences in the percentages of participants with explicit emotional and cognitive brand preferences.

The distribution of participants with a preference for Latvian or foreign foods, as measured by a demographic questionnaire (Preferred Foods), is not uniform. The preference for Latvian foods (23\%) is statistically insignificant with a small effect size $(h=0.20)$ exceeding the percentage of preferences for foreign food (15\%). The percentage of participants "without preferences" (62\%) is statistically significant, with a large effect size, exceeds the percentage of participants with a preference for both Latvian and foreign foods: $\varphi^{*}=6.57, p<0.001, h=0.81 ; \varphi^{*}=8.15, p<0.001, h=1.01$ accordingly.

Latvian foods are bought as often as foreign ones, yesterday-today $85 \%$ and $82 \%$ respectively. With the same price, $44 \%$ of participants buy Latvian foods and $23 \%$ - foreign ones. With the same quality, $45 \%$ buy Latvian foods and $21 \%$ - foreign ones. That is, according to both criteria, the percentage of purchases of Latvian foods is statistically significantly higher than the percentage of purchases of foreign 
foods $(p<0.001)$. The main reason for the change in preferences of Latvian foods to foreign ones is the quality of the foods (54\%) and experience (34\%).

From the results of all measurements carried out using the affective, cognitive and Self-concept IAT, it follows that the implicit preference is given to the Latvian food brand. The effect size is large.

It was not possible to find any important differences between the explicit preferences of Latvian and foreign brands.

This difference in the distribution of implicit and explicit attitudes towards the studied set of Latvian and foreign food brands can be explained by the fact that implicit attitudes towards brands change little over time. Explicit attitudes change faster. Some "equilibrium" in the distributions of the affective and cognitive components of explicit attitudes towards the proposed set of Latvian and foreign food brands can be explained as a consequence of the change in preferences for Latvian and foreign foods in general. The distribution of participants with a preference for Latvian or foreign foods, as measured by the demographic questionnaire (Preferred Foods), is not uniform. The preference for Latvian foods is still slightly higher than the preference for foreign foods. However, the percentage of participants "without preferences" greatly exceeds the percentage of participants with a preference for both Latvian and foreign foods. It also follows from the demographic questionnaire that Latvian foods are bought as often as foreign ones. At the same price and with the same quality, they buy more Latvian foods. The main reason for the change in preferences of Latvian foods to foreign ones is the quality of foods and experience.

In the present paper, the traditional model of information processing is adopted in the framework of two-process representation of attitudes. Its main postulates boil down to the adoption of a unified associative network as an important form of storing associative knowledge (spontaneous, automatic) in memory. This network interacts with a process based on propositional information processing processes, which are arbitrary (controlled), conscious and can, along with other cognitive processes, facilitate or limit the manifestation of associative processes. It is also customary to distinguish between the types of associations in accordance with the concept of the three-component structure of attitudes (affective, cognitive and behavioural). Moreover, an attempt was made to reveal the differences between the affective and cognitive (conceptual) bases of attitudes at the implicit and explicit levels of measurement, and the effects of the behavioural component are indirectly based on explicit assessments.

The issue of unitarity - plurality of specific associative networks encoding the types of affective, conceptual and behavioural information and their role in the formation of real behaviour is considered in the theoretical format of the promising neurocognitive Interactive Memory Systems Model (Amodio, 2019). Speaking from the perspective of this approach to understanding the memory system, it is important to assess the role of conceptual and instrumental levels of processing in the formation of consumer attitude assessments, which are based on independent, albeit situationally interacting, systems of representation of attitude associations - conceptual and instrumental. They correspond to declarative and procedural memory systems. In this paper, only some important aspects of this theory are touched upon.

Instrumental associations arise as a result of feedback that arises in the process of instrumental learning, which is active in nature and related experiences. This type of learning involves both making behavioural decisions and performing appropriate actions, and the process of positive or negative reinforcement as a result of these actions. Such learning more directly influences purposeful action (Samejima et al., 2005), which enhances the role of the effects of this learning in shaping real consumer decisions and behaviour in general.

All types of associations under consideration - conceptual, instrumental and affective - should be associated with the formation of behavioural decisions, but the instrumental associative network encoding the acquired experience of consuming the products of the respective brands presumably plays a dominant role.

The implicit and explicit methods for measuring attitudes used in this paper do not allow identifying specific associative processes and memory systems as understood by D.M. Amodio and colleagues, which underlie the development of responses to the tasks of the tests used. The results obtained also do not allow for a definite forecast of consumer behaviour when choosing foods of the brands under consideration in real life, i.e., to evaluate the effects of instrumental learning that is active in nature. However, these results provide some guidance for understanding the psychological mechanisms and systems that underlie the findings. 
It was found that direct explicit assessments of consumer preferences of Latvian or foreign brands did not reveal differences in the frequency of purchasing preferences for foods of these classes of brands. These assessments characterize the instrumental aspect of the explicit consumer attitude. Since at the behavioural level, the survey participants - consumers did not give a clear preference for the foods of Latvian brands, and all results obtained using the three IAT procedures indicate the implicit preference for Latvian brands, a contradiction arises. It can be resolved by assuming that the resulting differences between explicit assessments of consumer behaviour and implicit brand preferences were not associated with the influence of implicit instrumental associations encoding the effects of instrumental learning. Thus, the associative knowledge underlying the participants' responses to implicit measurement procedures was largely based on conceptual (cognitive) associations and affective implicit associations moderately associated with them. And the implicit instrumental network, a supposedly separate system (Amodio, 2019), was not affected by the procedures used.

This assumption needs to be clarified, considering the possibility of mixing affective and cognitive components of implicit attitudes, which may contradict each other, as a result of which it is impossible to predict consumer behaviour (Trendel, Werle, 2015).

In addition, it was found that the cognitive component makes the greatest contribution to the implicit assessment of attitude using the Self-concept IAT. This allows us to assume that this procedure is based mainly on the activation of cognitive (conceptual) associations that encode information about the price and quality of foods, but are not associated with the implicit instrumental network. All this emphasizes the role of the declarative rather than procedural memory system in the results obtained and illustrates the importance of the instrumental system noted by (Amodio, Berg, 2018) in understanding implicit attitudes and their influence on decision-making.

Cognitive (conceptual) implicit and explicit brand assessments also showed greater correspondence than the observed correspondence of affective assessments. This can be interpreted as evidence of a greater connection between conceptual systems that support implicit and explicit processes in the processing of attitudes than the corresponding affective processes suggest.

It was also found that the results of measurements of cognitive and affective components of brand attitudes by two different implicit procedures are closely related. Such a relationship reflects the difficulties (Trendel, Werle, 2015) noted in the literature in distinguishing these aspects of implicit attitude at the level of measurement, but it can also indicate a close interaction of the corresponding storage systems for relevant implicit information.

\section{Conclusions}

The aim of the study was achieved and the answers to the research questions were obtained.

The results of the research provide evidence in favour of the hypothesis of the independence of implicit and explicit "overall" attitudes, but on the example of attitudes toward food brands.

The expediency of separate considerations of the effects of conceptual and affective systems of attitude processing when measuring them to highlight the role of each of them in the processes of attitude processing has been confirmed. A separate analysis of these effects showed the importance of conceptual (cognitive) knowledge and a greater meaningful correspondence between the implicit and explicit levels of their representation, compared with the correspondence of these levels in the case of the affective system of attitude representation. By indirection, the results of the study are compatible with the concept of multiple systems for separate storage of information developed within the framework of the Multiple Memory Systems Model of Attitudes.

The discrepancy between the implicit assessments of brand attitudes and consumer behaviour declared by the participants raises the question of the need to study at the level of implicit measurement the role of instrumental associations and instrumental learning in the formation of brand attitudes. This study is promising for increasing the predictive (prognostic) validity of implicit procedures for measuring consumer attitudes and a better understanding of both their psychological nature and the mechanisms of functioning in real life. 


\section{Bibliography}

1. Amodio D.M. (2008). The social neuroscience of intergroup relations. European Review of Social Psychology, 19(1), 1-54. doi: 10.1080/10463280801927937

2. Amodio D.M. (2019). Social Cognition 2.0: An Interactive Memory Systems Account. Trends in Cognitive Sciences, 23(1), 21-33. doi: 10.1016/j.tics.2018.10.002

3. Amodio D.M., Berg J.J. (2018). Toward a Multiple Memory Systems Model of Attitudes and Social Cognition. Psychological Inquiry, 29(1), 14-19. doi: 10.1080/1047840X.2018.1435620

4. Amodio D.M., Devine P.G. (2006). Constructs and Unique Effects on Behavior. Journal of Personality and Social Psychology, 91(4), 652-661. doi: 10.1037/0022-3514.91.4.652

5. Amodio D.M., Harmon-Jones E., Devine P.G. (2003). Individual differences in the activation and control of affective race bias as assessed by startle eyeblink response and self-report. Journal of Personality and Social Psychology, 84, 738-753. doi: 10.1037/0022-3514.84.4.738

6. Amodio D.M., Mendoza S.A. (2010). Implicit intergroup bias: Cognitive, affective, and motivational underpinnings. In B. Gawronski, B.K. Payne (Eds.), Handbook of implicit social cognition: Measurement, theory, and applications. The Guilford Press, 353-374.

7. Amodio D.M., Ratner K.G. (2011). A Memory Systems Model of Implicit Social Cognition. Current Directions in Psychological Science, 20(3), 143-148. doi: 10.1177/0963721411408562

8. Bagozzi R.P., Burnkrant R.E. (1980). Single Component Versus Multicomponent Models of Attitude: Some Cautions and Contingencies for their Use. In J. Olson (Ed.), Advances in Consumer Research, 7. Ann Arbor: Association for Consumer Research, 339-344. Retrieved from https://www.acrwebsite.org/volumes/9694/volumes/v07/NA-07

9. Batra R., Ahtola O.T. (1990). Measuring the Hedonic and Utilitarian Sources of Consumer Attitudes. Marketing Letters, 2, 159-170. doi: 10.1007/BF00436035

10. Bosshard S.S., Bourke J.D., Kunaharan S., Koller M., Walla P. (2016). Established liked versus disliked brands: Brain activity, implicit associations and explicit responses. Cogent Psychology, 3(1), Article 1176691. doi: 10.1080/23311908.2016.1176691

11. Brunel F.F., Tietje B.C., Greenwald A.G. (2004). Is the Implicit Association Test a valid and valuable measure of implicit consumer social cognition? Journal of Consumer Psychology, 14(4), 385-404. doi: 10.1207/s15327663jcp1404_8

12. Chaiken S., Trope Y. (1999). Dual-process theories in social psychology. New York: Guilford Press.

13. Crites S.L.Jr., Fabrigar K.R., Petty R.E. (1994). Measuring the Affective and Cognitive Properties of Attitudes: Conceptual and Methodological Issues. Personality and Social Psychology Bulletin, 20(6), 619-634. doi: 10.1177/0146167294206001

14. Crowley A.E., Spangenberg E.R., Hughes K.R. (1992). Measuring the hedonic and utilitarian dimensions of attitudes toward product categories. Marketing Letters, 3, 239-249. doi: 10.1007/BF00994132

15. Cunningham W.A., Raye C.L., Johnson M.K. (2004). Neural correlates of evaluation associated with promotion and prevention regulatory focus. Cognitive Affective \& Behavioral Neuroscience, 5, 202-211. doi: 10.3758/CABN.5.2.202

16. Dalton P., Maute C., Oshida A., Hikichi S., Izumi Y. (2008). The Use of Semantic Differential Scaling to Define the Multi-Dimensional Representation of Odors. Journal of Sensory Studies, 23(4), 485-497. doi: 10.1111/j.1745-459X.2008.00167.x

17. Devine P. G. (1989). Prejudice and stereotypes: Their automatic and controlled components. Journal of Personality and Social Psychology, 56(1), 5-18. doi: 10.1037/0022-3514.56.1.5

18. Dijksterhuis Ap., Smith P.K., van Baaren R.B., Wigboldus D.H.J. (2005). The Unconscious Consumer: Effects of Environment on Consumer Behavior. Journal of Consumer Psychology, 15(3), 193-202. doi: 10.1207/s15327663jcp1503_3

19. Dimofte C.V. (2010). Implicit measures of consumer cognition: a review. Psychology and Marketing, 27(10), 921-937. doi: 10.1002/mar.20366

20. Dimofte C.V., Johansson J.K., Bagozzi R.P. (2010). Global Brands in the United States: How Consumer Ethnicity Mediates the Global Brand Effect. Journal of International Marketing, 18(3), 81-106. doi: 10.1509/jimk.18.3.81

21. Dimofte C.V., Johansson J.K., Ronkainen I.A. (2008). Cognitive and Affective Reactions of U.S. Consumers to Global Brands. Journal of International Marketing 16(4), 113-135. doi: 10.1509/jimk.16.4.113

22. Dimofte C.V., Yalch R.F. (2011). The mere association effect and brand evaluations. Journal of Consumer Psychology, 21(1), 24-37. doi: 10.1016/j.jcps.2010.09.005 
23. Eagly A.H., Chaiken S. (1993). The Psychology of Attitudes. New York: Harcourt Brace Jovanovich College Publishers.

24. Fazio R.H. (1990). Multiple processes by which attitudes guide behavior: The Mode Model as an integrative framework. Advances in experimental social psychology, 23, 75-109 doi: 10.1016/S00652601(08)60318-4

25. Fazio R.H. (1995). Attitudes as object-evaluation associations: Determinants, consequences, and correlates of attitude accessibility. In R.E. Petty, J.A. Krosnick (Eds.), Ohio State University series on attitudes and persuasion, Vol. 4. Attitude strength: Antecedents and consequences. Lawrence Erlbaum Associates, 247-282.

26. Fazio R.H., Jackson J.R., Dunton B.C., Williams C.J. (1995). Variability in automatic activation as an unobtrusive measure of racial attitudes: A bona fide pipeline? Journal of Personality and Social Psychology, 69(6), 1013-1027. doi: 10.1037/0022-3514.69.6.1013

27. Fishbein M.A., Ajzen I. (1975). Belief, attitude, intention, and behavior: An introduction to theory and research. Reading, MA: Addison-Wesley.

28. Friese M., Wänke M., Plessner H. (2006). Implicit consumer preferences and their influence on product choice. Psychology \& Marketing, 23(9), 727-740. doi: 10.1002/mar.20126

29. Gawronski B., Bodenhausen G.V. (2006). Associative and propositional processes in evaluation: An integrative review of implicit and explicit attitude change. Psychological Bulletin, 132(5), 692-731. doi: 10.1037/0033-2909.132.5.692

30. Greenwald A.G., Banaji M.R. (1995). Implicit social cognition: Attitudes, self-esteem, and stereotypes. Psychological Review, 102(1), 4-27. doi: 10.1037/0033-295X.102.1.4

31. Greenwald A.G., Farnham S.D. (2000). Using the Implicit Association Test to measure self-esteem and self-concept. Journal of Personality and Social Psychology, 79(6), 1022-1038. doi: 10.1037/00223514.79.6.1022

32. Greenwald A.G., McGhee D.E., Schwartz J.L.K. (1998). Measuring individual differences in implicit cognition: The Implicit Association Test. Journal of Personality and Social Psychology, 74(6), 1464-1480. doi: 10.1037/0022-3514.74.6.1464

33. Greenwald A.G., Poehlman T.A., Uhlmann E.L., Banaji M.R. (2009). Understanding and using the Implicit Association Test: III. Meta-analysis of predictive validity. Journal of Personality and Social Psychology, 97(1), 17-41. doi: 10.1037/a0015575

34. Herz M., Diamantopoulos A. (2013). Activation of country stereotypes: automaticity, consonance, and impact. Journal of the Academy of Marketing Science, 41, 400-417. doi: 10.1007/s11747-012-0318-1

35. Holbrook M.B., Hirschman E.C. (1982). The Experiential Aspects of Consumption: Consumer Fantasies, Feelings, and Fun. Journal of Consumer Research, 9(2), 132-140. doi: 10.1086/208906

36. Likert R. (1932). A technique for the measurement of attitudes. Archives of Psychology, 22, 1-55. Retrieved from https://legacy.voteview.com/pdf/Likert_1932.pdf

37. Maio G.R., Haddock G., Verplanken B. (2018). The psychology of attitudes and attitude change ( $3^{\text {rd }}$ ed.). London: Sage Publications.

38. Maison D., Greenwald A., Bruin R. (2001). The Implicit Association Test as a measure of implicit consumer attitudes. Polish Psychological Bulletin, 32(1), 61-79. Retrieved from https://faculty.washington.edu/agg/pdf/MaisonEtAl.PPB.final.pdf

39. Maison D., Greenwald A.G., Bruin R.H. (2004). Predictive validity of the Implicit Association Test in studies of brands, consumer attitudes, and behavior. Journal of Consumer Psychology, 14(4), 405-415. doi: 10.1207/s15327663jcp1404_9

40. Maison D., Gregg A. (2016). Capturing the consumer's unconscious: applying the implicit association test in consumer. In C.V. Jansson-Boyd, M.J. Zawisza (Eds.), The Routledge International Handbook of Consumer Psychology. London: Routledge Taylor \& Francis Group, 143-163.

41. Maison D., Maliszewski N. (2016). Worse but Ours," or "Better but Theirs?" The Role of Implicit Consumer Ethnocentrism (ICE) in Product Preference. doi: 10.3389/fpsyg.2016.01830

42. Millar M.G., Tesser A. (1986). Effects of Affective and Cognitive Focus on the Attitude - Behavior Relationship. Journal of Personality and Social Psychology, 51, 270-276.

43. Mitchell C.J., De Houwer J., Lovibond P.F. (2009). The propositional nature of human associative learning. Behavioural and Brain Sciences, 32(2), 183-198. doi: 10.1017/S0140525X09000855

44. Osgood C.E., Suci G.J., Tannenbaum P.H. (1957). The measurement of meaning. Urbana: University of Illinois Press. 
45. Payne B.K., Gawronski B. (2010). A history of implicit social cognition: Where is it coming from? Where is it now? Where is it going? In B. Gawronski, B.K. Payne (Eds.), Handbook of implicit social cognition: Measurement, theory, and applications. New York, NY: The Guilford Press, 1-15.

46. Perkins A., Forehand M. (2010). Implicit social cognition and indirect measures in consumer behavior. In B. Gawronski, B.K. Payne (Eds.), Handbook of implicit social cognition: Measurement, theory, and applications. New York, NY: The Guilford Press, 535-547.

47. Perkins A., Forehand M., Greenwald A.G., Maison D. (2008). The influence of implicit social cognition on consumer behavior: Measuring the non-conscious. In C. Haugtvedt, P. Herr, F. Kardes (Eds.), Handbook of Consumer Psychology. Hillsdale, NJ: Lawrence Erlbaum Associates, 461-475.

48. Petty R.E., Fazio R.H., Brinol P. (2009). Attitudes: Insights from the new implicit measures. New York: Psychology Press.

49. Plotka I., Igonin D., Blumenau N. (2016). Implicit Attitudes and Measurements: Effect of Context. International Business: Innovations, Psychology and Economics, 7, 2(12), 7-150. Retrieved from http://www.kuryba.lt/failai/zurnalai/2016_2.pdf\#page=7

50. Plotka I., Urbane B., Blumenau N. (2015). Explicit and Implicit Measurements of Brand Attitudes for Mobile Operators: Emotional and Cognitive Aspects. International Business: Innovations, Psychology, Economic, 6, 1(10), 45-60. Retrieved from http://www.kuryba.lt/en/failai/zurnalai/2015_1.pdf\#page=45

51. Priluck R., Till B.D. (2010). Comparing a customer-based brand equity scale with the Implicit Association Test in examining consumer responses to brands. Journal of Brand Management, 17, 413-428. doi: 10.1057/bm.2009.32

52. Rudman L.A. (2011). Implicit measures for social and personality psychology. London: Sage. doi: $10.4135 / 9781473914797$

53. Samejima K., Ueda Y., Doya K., Kimura M. (2005). Representation of action-specific reward values in the striatum. Science, 310, 1337-1340. doi: 10.1126/science.1115270

54. Sarabia-Andreu F., Sarabia-Sánchez F.J., Moreno-Albaladejo P. (2019). A New Attitudinal Integral-Model to Explain Green Purchase Intention. Sustainability, 11(22), 6290. doi: 10.3390/su11226290

55. Schnabel K., Asendorpf J.B., Greenwald A.G. (2008). Assessment of individual differences in implicit cognition: A review of IAT measures. European Journal of Psychological Assessment, 24, 210-217. doi: $10.1027 / 1015-5759.24 .4 .210$

56. Smith E.R., DeCoster J. (2000). Dual-process models in social and cognitive psychology: Conceptual integration and links to underlying memory systems. Personality and Social Psychology Review, 4(2), 108-131. doi: 10.1207/S15327957PSPR0402_01

57. Strack F., Deutsch R. (2004). Reflective and Impulsive Determinants of Social Behavior. Personality and Social Psychology Review, 8(3), 220-47. doi: 10.1207/s15327957pspr0803_1

58. Trendel O., Werle C.O.C. (2015). Distinguishing the affective and cognitive bases of implicit attitudes to improve prediction of food choices. Appetite, 104, 33-43. doi: 10.1016/j.appet.2015.10.005

59. Triandis H.C. (1977). Interpersonal Behavior. Monterey, CA: Brooks/Cole Publishing Co.

60. Voss K.E., Spangenberg E.R., Grohmann B. (2003). Measuring the Hedonic and Utilitarian Dimensions of Consumer Attitude. Journal of Marketing Research, 40(3), 310-320. doi: 10.1509/jmkr.40.3.310.19238 